\title{
Quality of life assessment in clinical research on Chinese medicine: Early experience and outlook
}

\author{
Lai Yi Eliza Wong' \\ Ping Chung Leung ${ }^{2}$ \\ 'Department of Community \\ and Family Medicine; ${ }^{2}$ Institute \\ of Chinese Medicine, School \\ of Public Health, The Chinese \\ University of Hong Kong, \\ Hong Kong, China
}

Correspondence: Ping Chung Leung Director, Center for Clinical Trials on Chinese Medicine, Institute of Chinese Medicine, The Chinese University of Hong Kong, 5/F, School of Public Health Building, Prince of Wales Hospital, Shatin, N.T., Hong Kong, People's Republic of China

Tel + I 85222528868

Fax + I 8522632544 I

Email: pingcleung@cuhk.edu.hk

\begin{abstract}
Patients' own account of the clinical progress is particularly important in situations of pain control, mental disturbances, and chronic problems. Chinese medicine does not directly target against a symptom or pathology, but emphasizes the maintenance of harmony between the vital forces of an individual. To achieve the harmony, usually long-termed treatment is required by consideration of the changing seasons and subject's constitution nature. With such unique requirements in Chinese medicine, the assessment of the quality of life becomes most important. There are obvious similarities between different systems of medical care. Hence the general domains of the quality of life should fit all patients, whether they are receiving conventional, modern medical care on alternative, Chinese medical care. Like different clinical research categories, specific areas (eg, cancer, women's problems) would need special additions of assessment. Chinese medicine is based on a uniquely different philosophy and the approach is not deductive, but individualized treatment is an essential requirement. The symptom/syndrome descriptions and interpretation are different from modern western medicine. Health-related quality of life is not sufficient for clinical trials using Chinese medicine, especially when Chinese medicine experts serve as chief investigators. Early attempts to develop an additional system to cover the need for Chinese medicine have been scanty. A lot of effort needs to be given before a practical instrument taking care of both the general domains of common interests and special feelings on health, relevant to Chinese medicine, could be established and made available. Users of Chinese medicine have to rely on generally acceptable yard-sticks with the addition of self-reporting symptoms unique to Chinese medicine.
\end{abstract}

Keywords: alternative medicine, Chinese medicine, quality of life

\section{Introduction}

We live in an era of the immense success of modern western medicine due to great advancements with the deductive approach. Every disease phenomenon is believed to be explainable and once a pathological process is clearly defined, usually the causation would be revealed and subsequently removed. In the world of modern clinical medicine, before they could be considered reliable, the ways which different pathologies can be removed are required to be supported by objective evidence and efficacy, as well as explanations of the biological mechanisms leading to the favorable outcome.

Modern medicine thus made impressive advances in the past decades via strict scientific approaches with stereotyped and innovative processes, which have been systematically developed and understood as a standard evidence-based medicine. Although the academics are aware of the many fallacies and imperfections when the modern practices and procedures are subjected to careful scrutiny, the scientists still insist that clinical practices should be required to go through the standard processes before they can properly be accepted as a recommended treatment modality. Standard assessment requirements have been created to evaluate the reliability of different means of clinical treatment. Thus in any clinical trial, a control group is considered 
mandatory, treatment and control groups are randomly selected, the control group takes placebo, and the results are analyzed with standard biostatistics methodology. Under those circumstances when the patients' own subjective feeling about the symptom changes need to be considered, those subjective data are considered to be of secondary importance, and are not considered reliable, since they are influenced by the patient's sociopsychological state of existence.

While clinicians and scientists may consider that only objectively measurable data are important, patients suffering from unmeasurable symptoms like pain, mental disturbances, and disabilities may disagree. This is the background in which psychiatrists and psychologists have started to advocate that what and how the patients perceived themselves should be respected as important factors in any organized treatment study and must also be given due attention.

The general domains of quality of life (QoL) were therefore developed as (WHOQOL) by the World Health Organization (WHO) in 1993 (WHO 1993). It consists of the following 6 categories:

1. Physical

- Pain and Discomfort

- Energy and Fatigue

- Sleep and Rest

2. Psychological

- Positive feeling

- Thinking, learning, concentration

- Self esteem

- Body image and appearance

- Negative feeling

3. Level of independence

- Mobility

- Activities of daily living

- Dependence on medication and treatment

- Working capacity

4. Social relationships

- Personal relationships

- Social support

- Sexual activity

5. Environment

- Physical safety and security

- Home environment

- Financial resources

- Health and social care: availability and quality

- Opportunities for new information and skills

- Participation in recreation and leisure

- Physical environment

- Transport

\section{Spirituality/religion/personal belief}

Another international generic QoL questionnaire, the Medical Outcomes Study Short Form 36-items Health Survey (SF36) was developed at the same time (Ware and Sherbourne 1992). It includes the following 2 categories:

1. Physical health

- Physical functioning

- Role-physical

- Bodily pain

- General health

2. Mental health

- Vitality

- Social functioning

- Role-emotional

- Mental health

In addition to the generic measure, disease-specific QoL questionnaire such as Pediatric Asthma Quality of Life (PAQOL), Menopause-Specific Quality of Life questionnaire (MENQOL), The European Organization for Research and Treatment of Cancer QOL-C30 (EORTC-QOLC30) were subsequently developed so as to further reflect patient's quality of life under different circumstances (Aaronson et al 1993; Juniper et al 1996; Yang et al 2005).

Folk medicine has developed from the understanding of disease process and symptom presentation which are closely linked with its unique culture and traditions. Assessment of the outcome of folk treatment therefore should include specific cultures and traditional ways of interpretation, which would contribute towards the considerations of changes in the QoL. Therefore, for clinical research on Chinese medicine, apart from the general qualitative assessments, special considerations need to be given to the assessment of the very specific Chinese medicine way of descriptions, and special measuring instruments need to be developed accordingly. Chinese medicine has a different philosophy from that of conventional medicine. The QoL of general physical or psychological states of existence are interpreted differently in Chinese medicine since QoL is closely correlated with the human constitution in Chinese medicine (Wang et al 2007). Take as an example, quality of sleep is interpreted as more complicated in Chinese medicine and includes the presence of dreams, palpitation during sleep, easiness of sleep at night, and the demand for sleep at daytime (Tam 1998). In therapeutics, western medicine has aimed at specificity, but Chinese medicine stresses the concept of the organism as a whole (Li 2006). Therefore, the domains and items of the QoL questionnaire used in modern western medicine may be not sensitive enough to reflect the efficacy of Chinese medicine 
on QoL. Actually, even for chronically ill patients, there are plenty of problems of their own that need to be addressed. Good examples include the following entities: cancer, allergy, degenerations, and aging problems. These examples obviously present with quite specific unique symptoms, which are not covered under the usual QoL instruments.

\section{Early experience}

Before a new instrument specific to Chinese medicine trials is ready, one has to rely on what is readily available, combining with simple inclusions of the assessment of aspects unique to Chinese medicine. Our experience of clinical trials where QoL assessment was involved is shown in Table 1.

In clinical studies where some symptoms could be considered essential, special items could be easily included to achieve a more comprehensive outcome which carries more meaning for both Chinese medicine practitioners and western medicine practitioners. Examples are given for herbal treatment study for viral respiratory infection (project 3 in Table 1). Our experience of exploring the QoL in clinical trial projects which either herbal treatment alone or integrated treatment was used has offered us insight into the feasibility of the existing instruments and the need for extensive future research.

In a study on allergic rhinitis using a herbal formula for treatment, we found it difficult to translate the terms of the Rhinoconjunctivitis QoL questionnaire developed by Meltzer (2001), which was considered standard for this allergic condition, therefore we hence resorted to SF36 alone. The deficiencies were immediately felt since the meaning of medical terms in modern medicine are different from the ones in Chinese medicine. In fact, translation could be simple and straightforward, short of validation. In a specific study on menopausal syndrome using herbal remedy (item 4 in Table 1), Haines and colleagues found that the specific MENQOL was conveniently translated and utilized, which gave very satisfactory results. Sung and colleagues felt that translation could be coupled with validation and this was done in our clinical trial on pediatric asthma using an innovative herbal formula (item 5 in Table 1). However, the interpretation of the term may be totally different in Chinese culture.

In our viral studies (item 2 and 3 in Table 1), the Vitality and Mental Health domains of the SF-36 QOL questionnaire

Table I Quality of life questionnaire used in clinical trials

\begin{tabular}{|c|c|c|c|}
\hline Project & Collaborator & $\begin{array}{l}\text { No of } \\
\text { patients }\end{array}$ & Quality of life questionanire \\
\hline \multicolumn{4}{|l|}{ Generic questionnaire } \\
\hline $\begin{array}{l}\text { I. Effects of Huangqi preparation } \\
\text { on diabetic foot ulcer (limb salvage) }\end{array}$ & Orthopedics and traumatology & 80 & $\begin{array}{l}\text { Medical Outcome Study Short Form 36-item Health } \\
\text { Survey (SF36) }\end{array}$ \\
\hline $\begin{array}{l}\text { 2. Effect of Chinese medicine } \\
\text { on the prevention of viral respiratory } \\
\text { infection in elderly }\end{array}$ & $\begin{array}{l}\text { Geriatric, chemical pathology, } \\
\text { elderly centre }\end{array}$ & 1480 & $\begin{array}{l}\text { Medical Outcome Study Short Form 36-item Health } \\
\text { Survey (SF36) }\end{array}$ \\
\hline $\begin{array}{l}\text { 3. Effect of Chinese medicine } \\
\text { on the prevention of viral respiratory } \\
\text { infection in healthy subject }\end{array}$ & Chemical pathology & 40 & $\begin{array}{l}\text { Medical Outcome Study Short Form 36-item Health } \\
\text { Survey (SF36) }\end{array}$ \\
\hline \multicolumn{4}{|l|}{ Disease-specific questionnaire } \\
\hline $\begin{array}{l}\text { 4. Effects of Danggui Buxue Tang } \\
\text { on menopausal symptoms }\end{array}$ & Obstetrics and gynecology & 100 & $\begin{array}{l}\text { Menopause Specific Quality of Life Questionnaire } \\
\text { (MENQOL) }\end{array}$ \\
\hline $\begin{array}{l}\text { 5. Effects of Chinese medicine } \\
\text { on pediatric asthma }\end{array}$ & Pediatrics & 100 & Paediatric Asthma Quality of life Questionnaire (PAQOL) \\
\hline $\begin{array}{l}\text { 6. Effects of Chinese medicine } \\
\text { on bone metastasis }\end{array}$ & Orthopedics and traumatology & 92 & $\begin{array}{l}\text { The European Organization for Research and Treatment } \\
\text { of Cancer QOL-C30 (EORTC-QOLC30) }\end{array}$ \\
\hline $\begin{array}{l}\text { 7. Effects of Chinese medicine } \\
\text { on colon cancer }\end{array}$ & Chemical pathology & 100 & $\begin{array}{l}\text { The Functional Assessment of Cancer Therapy General } \\
\text { Scale (FACT-G) }\end{array}$ \\
\hline 8. Effects of Chinese Medicine on Cough & $\begin{array}{l}\text { Community and family } \\
\text { medicine }\end{array}$ & 80 & Leicester Cough Questionnaire (LCQ) \\
\hline $\begin{array}{l}\text { 9. Effects of Chinese medicine on benign } \\
\text { prostatic hyperplasia }\end{array}$ & Surgery-urology & 20 & General Health Questionnaire 30 (GHQ30) \\
\hline
\end{tabular}


was used to assess the change in the general health-related QoL in the subject before and after taking Chinese medicine. Chinese medicine has a different philosophy from conventional medicine; SF-36, which was designed for the evaluation of conventional medical practice, therefore cannot be comprehensive and is unsuitable to cover all the changes expected from the Chinese medicine viewpoint. Health-related QoL specific to Chinese medicine should be supplemented to better validate the effect of Chinese medicine. From the above experiences, eight additional aspect changes were therefore added to supplement SF36 as follows:

1. Tongue sign (舌胎) reflecting appetite, etc...

2. Throat condition (咽喉) reflecting vitality, etc...

3. Bowel habit (大便習慣) reflecting body constitution, etc...

4. Stool condition (大便狀況) reflecting body constitution, etc...

5. Sleep pattern (睡眠) reflecting emotional status, etc...

6. Feeling of cold (寒) reflecting the balance of yin and yang, etc...

7. Feeling of hot (熱) reflecting the balance of yin and yang, etc...

8. Feeling of dampness (熱) reflecting the balance of yin and yang, etc...

Our clinical trials showed that the supplemented QoL items did not give extra difficulties in the data collection, and did give additional information which could facilitate to explore the efficacy of Chinese medicine.

Chinese medicine has a very strong cultural background, which leads not only to its popular use, but traditional beliefs also stand out strongly as supporting forces in the assessment processes of treatment efficacy. The choice not to give due respect and inclusion of the unique feelings and descriptions of the patient in their comprehensive medical assessment processes will be a mistake.

Nevertheless, there is a long way before a broadly applicable and easily adaptable instrument could be successfully developed. Chinese medicine remains very much an art of healing which refers to the difficulties in collecting objective data for efficacy assessment. When it is being used as a supportive agent, particularly in situations of chronic or palliative illness, obtaining objective data becomes even more difficult, and QoL naturally becomes essential. Chinese medicine mainly shows a performance-related efficacy which cannot be measured with simple objective parameters. The effect of traditional Chinese medicine mainly consists of whole effect, self-organization, self-stable model, holographic effect, and butterfly effect (Xie et al 2007). The pursuit of a QoL system which takes into considerations both the general universal requirements, together with specific inclusions of the special philosophical and cultural needs, would be fundamental for the further development of Chinese medicine. In this process of exploration and creation, a practical approach of selective inclusion and exclusion would be better than a full scale creation. Extreme metaphysical expressions might fail to find appropriate practical interpretations in a modern world. A realistic approach involving multiple collaborations between the two systems of philosophical approach is required.

\section{Discussion}

Chinese medicine deals with special symptoms and syndromes which carry special conceptual importance with reference to the Chinese traditional system of healing. How do these symptoms and syndromes respond to treatment carry special value for the Chinese medicine practitioner? Chinese medicine seldom deals with acute emergencies, but much more often deals with chronic sufferings. The description of the symptomatology is different from conventional modern medicine. Li (2006) observed that improving the QoL in patients with chronic diseases with integrated Chinese medicine and western medicine was better than that with western medicine alone or with Chinese medicine alone. The supplement of QoL assessment specific to Chinese medicine, therefore is important in its description as well as interpretation; not only should the boundaries be clinically comprehensive, but extra considerations should be given for the unique Chinese medicine concepts relevant to the particular disease entity. Zhao and colleagues (2005) stated that the assessment should include those special feelings of cold and heat, superficial and deep problems, false and real presentations, etc., which have been taken most seriously by traditional Chinese medicine experts for thousands of years.

As treatment is being given, the changes encountered in their subjective areas of feeling would need special records and instruments for measurement.

Assessing the changes in the QoL during treatment using Chinese medicine could go strictly according to the measuring instrument used for western medicine as just described, ie, accept a direct translation of the different domains. Alternatively, a better new system of measuring instrument could be developed basing more on the basic Chinese medicine philosophy.

The WHO in 1993 defined QoL as an individual's own perception of his position in life in the context of the culture and value system in which he lives and in relation 
to his goals, expectation standards, and concerns (WHO 1993). This concept could be kept and the emphasis on the respect to one's culture and value system well supports the need to develop a special measuring instrument for Chinese medicine.

Zhao and colleagues (2005), and Zhao and Chan (2003, 2005) attempted to introduce the Chinese medicine concept into the overall consideration of QoL. Zhao and Chan (2005), and Ware and Sherbourne (1992) explained that the fundamental concept of healing in Chinese medicine was built on the harmonization of opposing forces, which not only included the individual's biological state, but also his relationship with Nature and society. This idea could be expressed as shown in Figure 1.

The harmonization of one's physical and mental state in the Chinese medicine context deserves more complicated research. In more traditional technology relevant to Chinese medicine, the balance or harmonization of the physique and vitality involves multiple measurements which include:

1. Physique

- General body state

- Physiological manifestations

- Appetite

- Excretion (bowel and urine)

- Physical strength

- Sexual strength

- Demand on respiratory push

- Demand on rest (sleep)
2. Vitality

- Spiritual confidence

- Cognitive ability

- Linguistic expression

- Visual expression

The harmonization principle, balanced between physique and vitality, serves as the big umbrella under which different manifestations are explored. This exploration should include what Chinese medicine clinicians consider as important reactions of the individual to their own physiological and psychological states as well as to social and natural environments. The seven emotions in traditional Chinese medicine form the main contents of the individual's reactions: joy, anger, worry, pensiveness, grief, fear, and fright (Tam 1998).

Based on the principles described above, a new QoL questionnaire could be constructed and tested on a series of clinical responses while treatment is being given (Leung 2003). The more comprehensive construct would allow a better prospect of mutual respect and collaboration between the western practitioners and Chinese medicine practitioners. Besides, there are a number of studies have revealed that supplemented Chinese medicine-specific QoL items were strongly correlated to WHOQOL-100 and SF-36 and also showed the unique efficacy of Chinese medicine on the quality of life (Zhao et al 2006). In the meantime the authors wish that the Chinese medicine practitioner would continue on their serious exploration and development of their measuring yard-stick which could then be quantified and thence

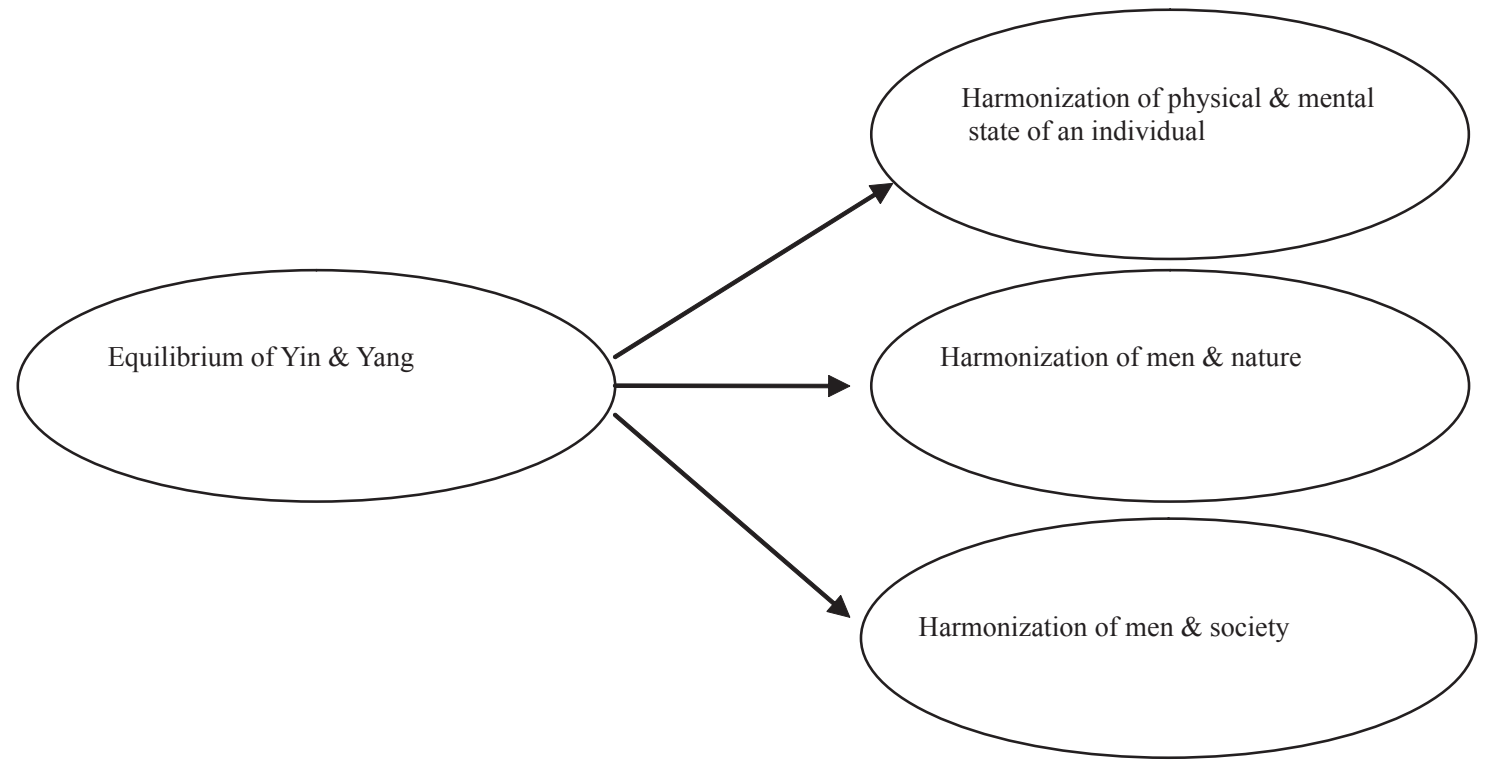

Figure I Fundamental concept of healing in Chinese medicine built on harmonization and equilibrium. 
acceptable to all clinicians as assessment instruments, which must be acceptable to both western and Chinese medicine practitioners and be uncomplicated.

We are aware of a study being carried out in the United States of America for the development of a Chinese Medicine Assessment Measure using an interdisciplinary approach and relying on the Delphi method (Schnyer et al 2005). In this study, the diagnostic framework and clinical reasoning of traditional Chinese medicine was given full recognition and a structured assessment instrument was developed for use in clinical trials of acupuncture and possibly for other Chinese medicine therapies later. The instrument, labeled as Traditional East Asian Medicine Structured Interview, Traditional Chinese Medicine version, (TEAMSI-TCM) was created and was being validated. This must be an ambitious attempt and we look forward to further reports. The degree of complexity involved, however, appears to be worrying.

\section{Acknowledgments}

The authors report no conflicts of interest in this work.

\section{References}

Aaronson NK, Ahmedzai S, Bergman B, et al. 1993. The European Organization for Research and Treatment of Cancer QLQ-C30: a quality-of-life instrument for use in international clinical trials in oncology. J Natl Cancer Inst, 85:365-76.

Juniper EF, Guyatt GH, Feeny DH, et al. 1996. Measuring quality of life in children with asthma. Qual Life Res, 5:35-46.

Leung PC. 2003. Clinical trials using Chinese medicine. In: Leung PC, Xue CC, Cheng YC (Eds.). A Comprehensive Guide to Chinese Medicine. NJ: River Edge, World Scientific Publisher.
Li XJ. 2006. Effect of integrated Chinese and western medicine in treating diabetes mellitus with the quality of life criterion. Chinese $J$ Clin Rehabilit, 10:154-61.

Meltzer EO. 2001. Quality of life in adults and children with allergic rhinitis. Effect of integrated Chinese and western medicine in treating diabetes mellitus with the quality of life criterion. J Allergy Clin Immunol, 180:945-53.

Schnyer RN, Conboy LA, Jacobson E, et al. 2005. Development of a Chinese medicine assessment measure: an interdisciplinary approach using the Delphi method. J Altern Complement Med, 11:1005-13.

Tam K. 1998. Theory of Chinese Medicine. Taiwan: Cheng Chung Book Co. Ltd.

Wang Q, Zhu YB, Wu CY, et al. 2007. Correlation between constitution in Chinese medicine and health-related quality of life. Journal of Clinical Rehabilitative Tissue Engineering Research, 11:9946-50.

Ware JE Jr., Sherbourne CD. 1992. The MOS 36-item short-form health survey (SF-36). I. Conceptual framework and item selection. Med Care, 30:473-83.

[WHO] World Health Organization. 1993. The development of the WHO quality of life assessment instrument. Geneva: World Health Organization.

Xie RM, Du BX, Huang Y, et al. 2007. Considerations on intervention goal and efficacy evaluation of traditional Chinese medicine in the treatment of neurological diseases. Chin J Integr Med, 13:148-51.

Yang H, Cheng F, Wang X, et al. 2005. [On clinical application and assessment of Menopause-Specific Quality of Life Questionnaire.] Zhonghua Liu Xing Bing Xue Za Zhi, 26:47-50.

Zhao L, Chan K. 2003. Quality of Life Assessment: An Outcome estimation of Chinese Medical Treatment. Chin J Integr Med, 9:299-301.

Zhao L, Chan K. 2005. Building a bridge for integrating Chinese medicine into conventional health care: Observations drawn from the development of the Chinese Quality of Life Instrument. Am J Chin Med, 33:897-902.

Zhao L, Chan K, Leung KF, et al. 2005. The conceptual framework of the Chinese Quality of Life (ChQOL) instrument. Ann Trad Chin Med, 1:187-95.

Zhao L, Liu FB, Leung KF, et al. 2006. Reliability and validity of the Chinese quality of life instrument. Chin J Clin Rehabilit, 10:1-3. 